von 1992 leider wenig geändert. Familie und Beruf sind vereinbar geworden, nicht aber Familie und Karriere. Dies wirft die Frage auf, was der DRB in den mehr als 20 Jahren gemacht hat. Wäre es nicht auch seine Aufgabe gewesen, auf die Beseitigung der Hindernisse hinzuwirken? Der DRB, ein männlich geprägter Verband, hat die Benachteiligung der Kolleginnen beim beruflichen Aufstieg im Wesentlichen ausgeblendet. Die althergebrachten Beförderungsstrukturen und das nicht seltene Vorurteil von Personalverantwortlichen, Mütter hätten kein Interesse am beruflichen Fortkommen, haben den Kollegen Konkurrentinnen vom Hals gehalten. Der Richterbund mag einwenden, die betroffenen Frauen hätten sich doch im Verband in ihrem Sinne engagieren können. Theoretisch ja, praktisch hatten und haben sie aber in der Regel keinen Partner zuhause, der ihnen den Rücken für Verbandsaktivitäten neben dem Beruf und der Familie frei hält. Die Mitarbeit im DRB ist zweifelsohne karriereförderlich. Wenn eine Frau mittut, wird in der Regel etwas aus ihr und das war es dann in Sachen Frauenförderung.

Der Vorwurf fehlenden Engagements hat allerdings einen wahren Kern: Frauen unterschätzen den Nutzen von beruflichen Netzwerken für ihr Fortkommen (Brückner, djbZ 2013, 191). Leistung und Befähigung sind eine Grundvoraussetzung für Erfolg, sie reichen aber meist nicht, weil Menschen mit eigenen Interessen darüber entscheiden, wer befördert wird. Daher ist es wichtig, Schaltstellen zu erkennen und zu nutzen, die Einfluss auf die eigene Karriere haben. Dazu zählen nicht nur Sonderverwendungen als Präsidialrichter/in und Pressesprecher/ in, sondern auch als Gleichstellungsbeauftragte und ein Engagement in Berufsverbänden. Die örtlichen Verbände des DRB verfügen meist dank ihrer hervorragenden Vernetzung in die Gerichts- und Behördenleitungen über einen sehr guten Einblick in und Einfluss auf die oft verschlungenen Wege, wer wann welche Karriere macht. Kontakte u.a. zu den Führungsspitzen der Justiz können über den djb geknüpft werden, der zudem Frauen bei der Besetzung von Top-Positionen ins Gespräch bringt. Schließlich sollten Richterinnen und Staatsanwältinnen das Amt der Gleichstellungsbeauftragten nicht unterschätzen und für ihre Belange nutzen. Denn es verschafft umfassende Insider-Kenntnisse und gibt das Recht, selbst initiativ zu werden (Schübel, djbz 2013, 86).

Wir müssen keine neuen Wege suchen, sondern uns die vorhandenen Pfade erschließen - frei nach dem Spruch von Simone de Beauvoir, den der Interministerielle Arbeitskreis der Gleichstellungsbeauftragten im Bund propagiert: „Frauen, die nichts fordern, werden beim Wort genommen. Sie bekommen nichts. “

\title{
European Women Shareholders Demand Gender Equality: Erstes Projekttreffen in Brüssel
}

Das djb-Projekt „European Women Shareholders Demand Gender Equality " hat im April 2014 von der Europäischen Kommission neben 16 weiteren europäischen Projekten den Zuschlag für eine Förderung nach dem PROGRESS-Programm erhalten. Es wird kofinanziert vom Bundesministerium für Familie, Senioren, Frauen und Jugend (BMFSFJ), dem Ministerium für Justiz und Gleichstellung des Landes Sachsen-Anhalt und der Finnischen Handelskammer. Am 16. Mai 2014 war offizieller Projektbeginn.

Am 19. Juli war es endlich soweit: Das djb-Projektteam, nationale Koordinatorinnen und Vertreterinnen der Projektpartner trafen sich erstmals im Rahmen des neuen djb-Projekts „European Women Shareholders Demand Gender Equality“. Ort des

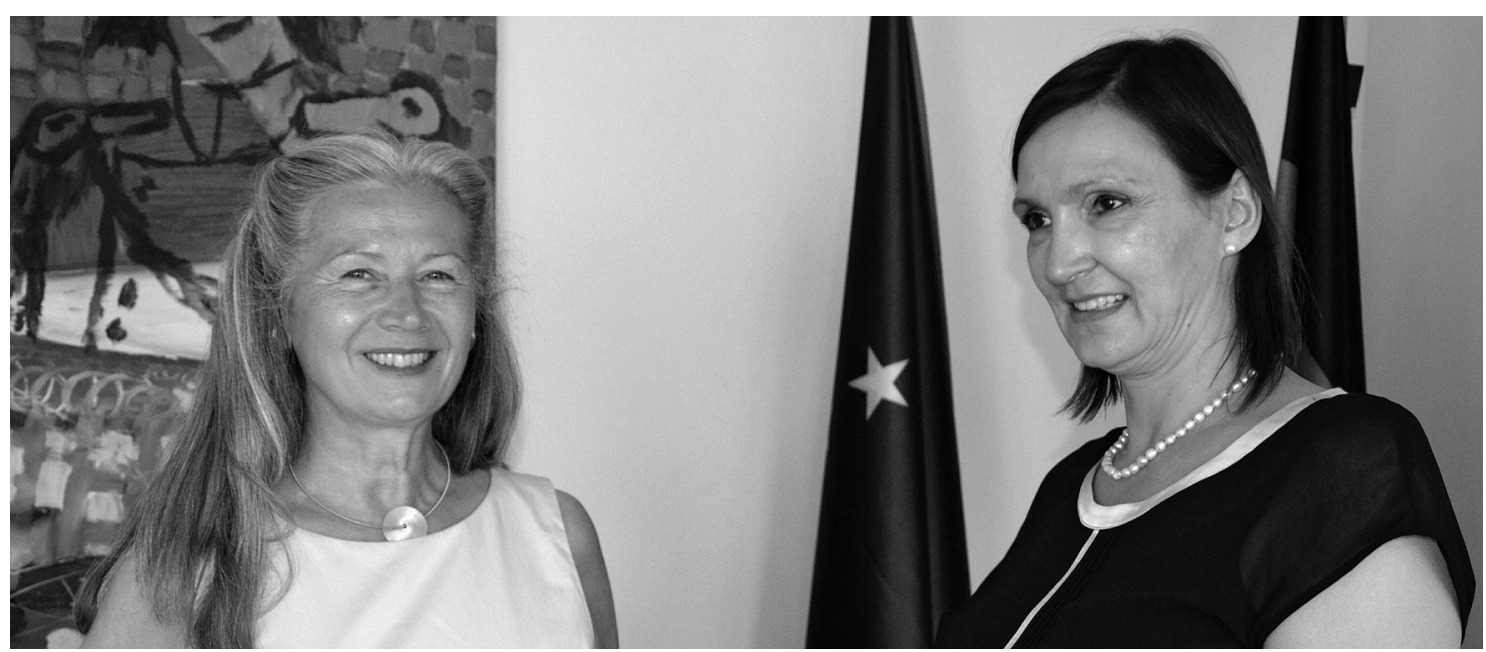

$\Delta$ Ramona Pisal, djb-Präsidentin, und Prof. Dr. Angela Kolb, Ministerin für Justiz und Gleichstellung des Landes Sachsen-Anhalt (Foto: djb)

Miruna Bucurescu, Linda Walczak Projektmitarbeiterinnen, Deutscher Juristinnenbund e.V., Berlin 


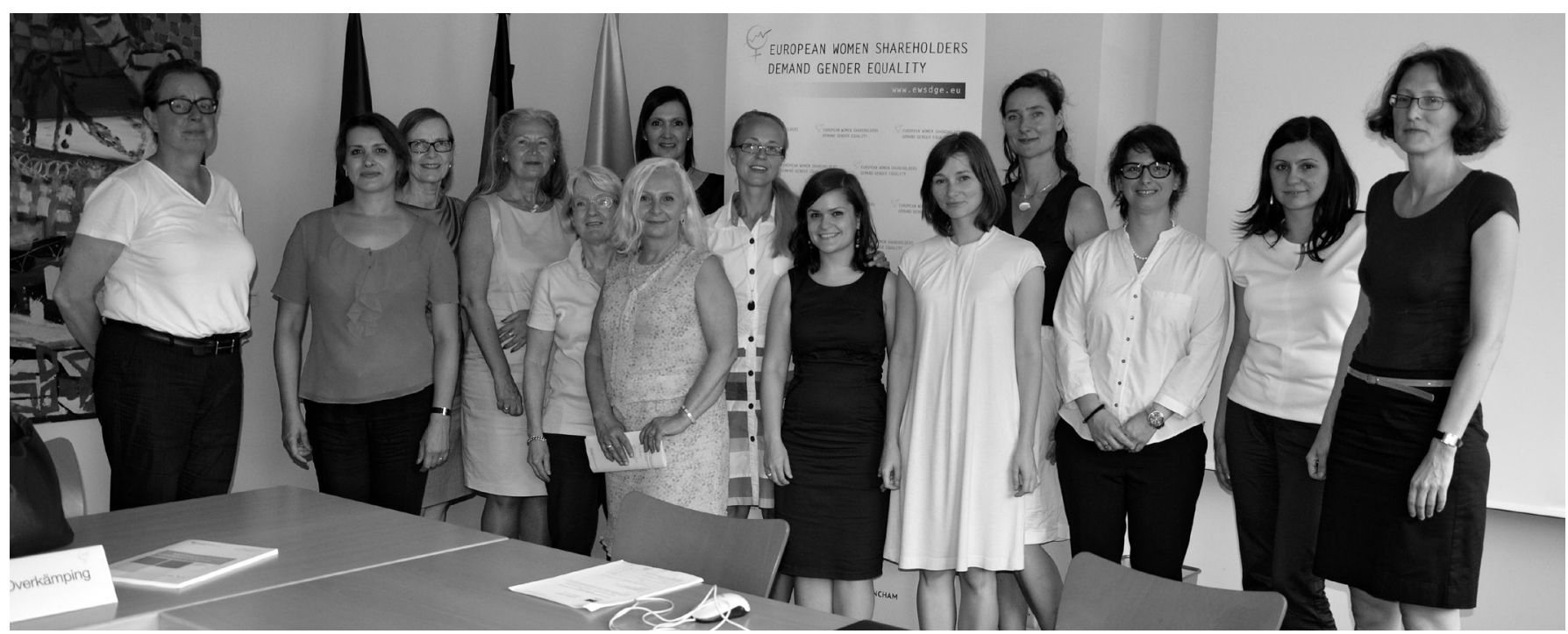

$\Delta$ Erfolgreiche, europäische Zusammenarbeit: Die Teilnehmerinnen des ersten EWSDGE Projekttreffens: Sabine Overkämping, Vorsitzende der djb-Kommission Öffentliches Recht, Europa- und Völkerrecht; Svetlana Ganeva, nationale Koordinatorin Bulgarien; Margarete Hofmann, djb-Vizepräsidentin; Ramona Pisal, djb-Präsidentin; Dr. Annette Matthias, nationale Koordinatorin BENELUX; Ministerin Prof. Dr. Angela Kolb, Projektpartner Ministerium für Justiz und Gleichstellung des Landes Sachsen-Anhalt; Dr. Katalin Prandler, nationale Koordinatorin Ungarn; Katharina Miller, nationale Koordinatorin Spanien; Miruna Bucurescu, Projektmitarbeiterin, Linda Walczak, Projektmitarbeiterin; Sylvia Cleff Le Divellec, nationale Koordinatorin Frankreich; Elsa Papadopoulou, EWLA-Mitglied; Kamelia Angelova, nationale Koordinatorin Bulgarien; Dr. Henrike Franz, Leiterin der Vertretung des Landes Sachsen-Anhalt in Brüssel und Gastgeberin des Projektstreffens (v.l.n.r., Foto: djb)

Treffens war die Vertretung des Landes Sachsen-Anhalt bei der Europäischen Union in Brüssel. Im Anschluss an eine erste Vorstellungsrunde der insgesamt 17 aus ganz Europa angereisten Beteiligten, in der Erwartungen und Vorstellungen ausgetauscht wurden, folgte ein intensiver Arbeitstag.

Europaweiter Einsatz für die Erhöhung des Frauenanteils in Führungspositionen lautet das Ziel des Projektes. In diesem Sinne sollen von Januar bis Juli 2015 die Hauptversammlungen von bis zu 100 börsennotierten Unternehmen, darunter die aller 50 EURO STOXX-Unternehmen besucht werden. Die rechtlichen Grundlagen, die eine Teilnahme an der Hauptversammlung eines börsennotierten Unternehmens ermöglichen, bildeten daher einen der Schwerpunkte des Treffens. Bereits gemachte Erfahrungen, wie in Deutschland und Spanien, sowie mögliche Herausforderungen in den anderen Ländern wurden unter den Teilnehmerinnen intensiv diskutiert.

Des Weiteren wurde ein Fragenkatalog entwickelt, der als Grundlage für die Hauptversammlungsbesuche der europäischen Unternehmen dienen soll. Das europapolitische Fundament des Fragenkatalogs bildet dabei der Kommissionsvorschlag für eine Aufsichtsrätinnen-Richtlinie zur Einführung einer Zielquote für Frauen in Aufsichtsräten von börsennotierten europäischen Unternehmen bis 2020 von 40 Prozent $^{1}$, sowie die bereits vom Europäischen Parlament verabschiedete und die vom Rat noch zu verabschiedende CSR-Richtlinie ${ }^{2}$. Diese enthält konkrete Vorgaben zu Diversity und Chancengleichheit in Führungspositionen und verpflichtet europäische Unternehmen zukünftig in ihrem Unternehmensbericht Angaben zu Alter, Geschlecht, Ausbildung und beruflichem Hintergrund ihres Führungspersonals zu machen. Darüber hinaus müssen sie Auskunft zu den Zielen, Maßnahmen und Ergebnissen ihrer Diversity-Politik geben. Tun sie dies nicht, muss dies im Bericht begründet werden. Auf beide Richtlinien, die auf eine Erhöhung des Frauenanteils in Führungspositionen auf europäischer Ebene angelegt sind, wird im Fragenkatalog Bezug genommen werden. Weitere Fragen, die auf den Hauptversammlungen im Rahmen des Projekts an die Vorstände und Aufsichtsräte gestellt werden, beziehen sich auf die Rekrutierung von Frauen für höhere Führungspositionen. Dabei geht es zum einen um Personalentwicklungskonzepte und Unternehmensstrategien, zum anderen um die Angabe von konkreten Zahlen.

Als nächster Schritt ist im Herbst ein Workshop für alle nationalen Koordinatorinnen in Helsinki in der finnischen Handelskammer, einem weiteren Projektpartner, geplant. Die finnische Erfahrung mit der erfolgreichen Erhöhung des Anteils von Frauen in der Privatwirtschaft soll im Sinne von vorbildlicher Praxis genutzt werden. Darüber hinaus dient der Workshop dem Coaching der nationalen Koordinatorinnen, deren maßgebliche Aufgabe im Rahmen des Projekts darin besteht, die Hauptversammlungsbesuche in ihrem Land zu organisieren und auszuwerten.

Im Anschluss werden die nationalen Koordinatorinnen wiederum Workshops in ihren eigenen Ländern durchführen, um die zukünftigen Rednerinnen und Dokumentalistinnen auf den Hauptversammlungen bestens auf ihre Aufgabe vorzubereiten.

1 Europäische Kommission: Vorschlag für eine Richtlinie des Europäischen Parlaments und Rates zur Gewährleistung einer ausgewogeneren Vertretung von Frauen und Männern unter den nicht geschäftsführenden Direktoren/Aufsichtsratsmitgliedern börsennotierter Gesellschaften und über damit zusammenhängende Maßnahmen. $\operatorname{COM}(2012)$ 614, 14.11.2012.

2 Europäische Kommission: Vorschlag für eine Richtlinie des Europäischen Parlaments und Rates zur Änderung der Richtlinien 78/660/EWG und 83/349/EWG des Rates im Hinblick auf die Offenlegung nichtfinanzieller und die Diversität betreffender Informationen durch bestimmte große Gesellschaften und Konzerne, $\operatorname{COM}(2013)$ 207, 16.4.2013. 
Die Unternehmen, deren Hauptversammlungen im kommenden Jahr besucht werden, sind zum einen alle Unternehmen aus dem EURO STOXX 50. Dieser Aktienindex beinhaltet die wichtigsten und größten Unternehmen der Eurozone und berücksichtigt acht in das Projekt einbezogene Länder: Belgien, Frankreich, Deutschland, Irland, Italien, Luxemburg, Niederlanden und Spanien. Weitere bis zu 50 Unternehmen befinden sich in Bulgarien, Ungarn und dem Vereinigten Königreich. Da die Unternehmen dieser Länder nicht im EURO STOXX vertreten sind, werden hier die Hauptversammlungen von Unternehmen aus den jeweils nationalen Aktienindizes - SOFIX, BUX und FTSE 100 - besucht.

Eine gewisse Kontinuität bedeutet die Teilnahme an den Hauptversammlungen der deutschen Unternehmen, die im EURO STOXX 50 geführt werden. Im Rahmen des mittlerweile abgeschlossenen djb-Projektes „Aktionärinnen fordern Gleichberechtigung “ (2009-2013) wurden bereits alle Unternehmen mehrfach besucht. 2015 wird der djb erneut auf den Hauptversammlungen der folgenden 14 Unternehmen vertreten sein: Allianz, BASF, Bayer, BMW, Daimler, Deutsche Bank, Deutsche Post, Deutsche Telekom, E.ON, Münchener Rück, RWE, SAP, Siemens, Volkswagen.

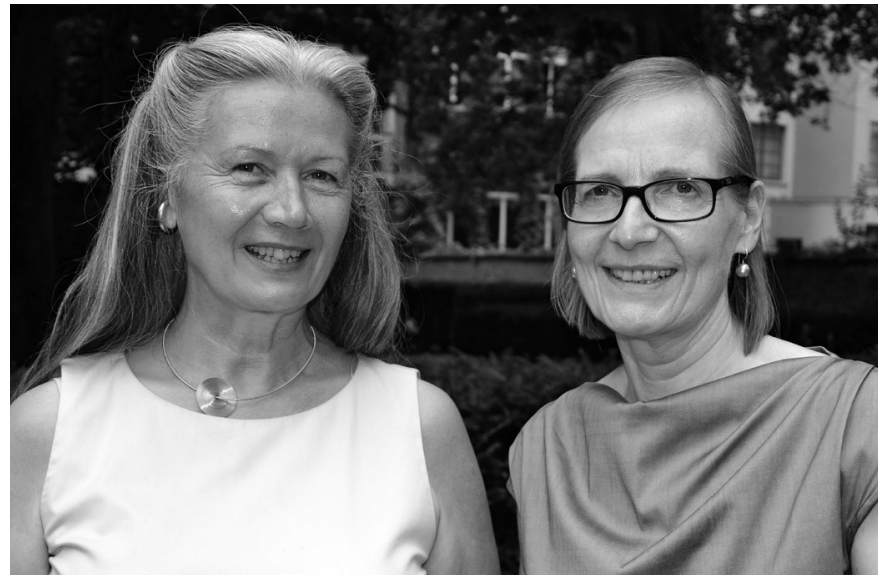

A Ramona Pisal, djb-Präsidentin, und Margarete Hofmann, djb-Vizepräsidentin (Foto: djb)

Um das Projekt erfolgreich durchzuführen, begrüßen wir jede Form der Unterstützung. Wir freuen uns über die Übertragung von Stimmrechten eigener Aktien und über ehrenamtliche Teilnehmerinnen an den Hauptversammlungen. Wenn Sie Interesse an einer Mitarbeit haben, setzten Sie sich mit dem Projektbüro in Verbindung: eu-womenshareholders@djb.de

\section{Karriere-Sprungbrett Frauenverband?}

\section{Seine Funktion als ,Motor gesellschaftlicher Veränderung“}

Welche Rolle spielen Frauenverbände ${ }^{1}$ bei der Karriereförderung und inwiefern wirkt sich die Mitgliedschaft in einem Frauenverband karrierefördernd aus? Ausgehend von dieser Fragestellung wurde in den vergangenen drei Jahren ein Projekt in der Stiftung Archiv der deutschen Frauenbewegung durchgeführt, dessen Abschlusspublikation „Karrieren von Frauen sind Drahtseilakte. Frauenverbände als Netzwerke der Karriereförderung “ (2014) nun im Verlag Barbara Budrich erschienen ist.

Die Autorinnen sind, Helke Dreier, Historikerin M.A. und wissenschaftliche Mitarbeiterin in der Stiftung, und Dr. Regina Löneke, Kulturanthropologin. Ihr Ziel war es herauszuarbeiten, ,in welchem Zusammenhang Ehrenamt, Beruf und Karriere stehen, wie Frauen beruflich von ihrer Mitgliedschaft in einem Frauenverband profitieren und inwiefern die Berufs- bzw. Karriereförderung ihrer Mitglieder Teil des Aufgabenspektrums der untersuchten Frauenverbände ist" (11). Zielgruppe des Projekts sind junge Frauen, wobei die Autorinnen ausdrücklich auf die verwendete, weite Definition des Begriffes hinweisen. Sie verstehen darunter „nicht das Lebensalter, sondern eine Lebensphase im Erwerbsleben“ (17), die aufgrund der Vergleichbarkeit der beruflichen Situation gleichermaßen sowohl Berufseinsteigerin- nen als auch Berufsrückkehrerinnen berücksichtigt. Insgesamt wurden 13 Frauenverbände ${ }^{2}$ in Deutschland hinsichtlich ihrer Möglichkeiten zur Karriereförderung ihrer Mitglieder und 48 Verbandsfrauen anhand von Expertinneninterviews untersucht. Auch der Deutsche Juristinnenbund e.V. (djb) und vier seiner Mitglieder beteiligten sich an dem Projekt.

Anhand des gewählten Ansatzes der Autorinnen ergibt sich eine zweifache Perspektive, die eine umfassende und kritische Hinterfragung ermöglicht, „inwiefern sich das aktive Engagement von Frauen in einem Frauenverband beruflich positiv oder negativ auswirkt" (16). Zum einen werden auf der individuellen Ebene die Frauen als Mitglieder von

1 Der Begriff subsumiert im Folgenden Frauenvereine, Berufsverbände, Verbände mit konfessionellen oder sozialen Auftrag etc. Vgl. auch insbesondere S. 28-32.

2 Bundesverband der Frauen in Business und Management e.V. (B.F.B.M.), Bundesverband der Migrantinnen in Deutschland e.V., Demokratischer Frauenbund e.V. (dfb), Deutscher Ärztinnenbund e.V. (DÄB), Deutscher Evangelischer Frauenbund e.V. (DEF), Frauen in Naturwissenschaft und Technik e.V. (NUT), deutscher ingenieurinnenbund e.V. (dib), Deutscher Juristinnenbund e.V. (djb), Deutscher LandFrauenverband e.V. (dlv), Journalistinnenbund e.V. (JB), Katholischer Deutscher Frauenbund e.V. (KDFB), Verband medizinischer Fachberufe e.V. (vmf), Weibernetz e.V.
Katrin Lange, Mitarbeiterin, Deutscher Juristinnenbund e.V., Berlin 